drug combinations that can simultaneously target the undifferentiated leukemic cell populations as well as the more mature myeloid lineages (especially monocytes) may represent a powerful way to prioritize the most promising drug combinations for pre-clinical study and for clinical development. Clearly, this report demonstrates just the beginning of utility of this exciting drug screening platform.

\section{References}

1. Friedman AA, Letai A, Fisher DE, Flaherty KT. Precision medicine for cancer with next-generation functional diagnostics. Nat Rev Cancer. 2015;15(12):747-756.

2. Rossi DJ, Jamieson CH, Weissman IL. Stems cells and the pathways to aging and cancer. Cell. 2008;132(4):681-696.

3. Majumder MM, Leppa AM, Hellesoy M, et al. Multi-parametric single cell evaluation defines distinct drug responses in healthy hematological cells that are retained in corresponding malignant cell types. Haematologica. 2020;105(6):1527-1538.

4. Pemovska T, Kontro M, Yadav B, et al. Individualized systems medicine strategy to tailor treatments for patients with chemorefractory acute myeloid leukemia. Cancer Discov. 2013;3(12):1416-1429.

5. Lagadinou ED, Sach A, Callahan K, et al. BCL-2 inhibition targets oxidative phosphorylation and selectively eradicates quiescent human leukemia stem cells. Cell Stem Cell. 2013;12(3):329-341.

6. Kuusanmaki H, Leppa AM, Polonen P, et al. Phenotype-based drug screening reveals association between venetoclax response and differentiation stage in acute myeloid leukemia. Haematologica. 2020;105(3):708-720.

7. Pei S, Pollyea DA, Gustafson A, et al. Monocytic Subclones Confer Resistance to Venetoclax-Based Therapy in Patients with Acute Myeloid Leukemia. Cancer Discov. 2020;10(4):536-551.

\title{
To target the untargetable: elucidation of synergy of APR-246 and azacitidine in TP53 mutant myelodysplastic syndromes and acute myeloid leukemia
}

\author{
David A. Sallman
}

\author{
Department of Malignant Hematology, H. Lee Moffitt Cancer Center, Tampa, FL, USA \\ E-mail: DAVID A. SALLMAN - david.sallman@moffitt.org
}

doi:10.3324/haematol.2020.249060

M utations of the tumor suppressor gene TP53 represent a common mutation in myeloid malignancies, occurring in 10-20\% of patients with de novo myelodysplastic syndromes (MDS) and acute myeloid leukemia (AML) with profound negative impact on outcomes and a median overall survival (OS) of 6-12 months. ${ }^{1 .}$ ${ }^{3}$ Critically, the clonal burden of TP53, that is the variant allele frequency (VAF) and/or allelic state of TP53, is intimately tied with the clinical trajectory of these patients and is a robust, independent predictor of survival. ${ }^{47}$ Given the poor OS and lack of therapeutic options for TP53 mutant MDS/AML patients, a number of novel agents are being investigated in this patient group. ${ }^{8}$ Of these, APR246 has evoked considerable excitement based on its robust clinical efficacy in combination with azacitidine in TP53 mutant MDS/AML patients. ${ }^{9,10}$ In this issue of Haematologica, ${ }^{11}$ Maslah et al. describe compelling preclinical synergy of APR-246 in combination with azacitidine in TP53 mutated MDS and AML and, more importantly, identify a novel molecular mechanism underlying the observed synergy.

Recent elegant work has definitively identified that TP53 missense mutations in myeloid malignancies result in a dominant-negative effect without evidence of neomorphic gain-of-function activities, ultimately leading to a selection advantage when exposed to DNA damage. ${ }^{12}$ Thus, restoring wild-type function in TP53 mutant clones would be of profound beneficial impact. APR-246, a methylated PRIMA-1 analog, is a novel, first-in-class, small molecule that selectively induces apoptosis in TP53 mutant cancer cells. Mechanistically, APR-246 is spontaneously converted into the active species methylene quinuclidinone (MQ), which is able to covalently bind to cysteine residues in mutant p53 thereby producing thermo- dynamic stabilization of the protein and shifting equilibrium toward a functional conformation. ${ }^{13,14}$ APR-246 monotherapy was originally investigated in a phase I trial including AML patients with clinical activity and correlative data identifying activation of p53-dependent pathways. ${ }^{15,16}$

Maslah et al. identified in TP53 mutant cell lines, in vivo models, and primary patient samples that the combination of APR-246 and azacitidine results in a synergistic pro-apoptotic effect as well as a dramatic reduction in cell proliferation via cell cycle arrest (Figure 1). As the majority of TP53 mutations are missense and located in the DNA binding domain, synergy experiments were performed with the SKM1 cell line, which harbors a homozygous hotspot mutation of TP53 (p. R248Q), and thus is an appropriate representation of clinical disease. ${ }^{17}$ Combination therapy of APR-246 and azacitidine resulted in a doubling of apoptotic cells versus azacitidine alone as well as $83 \%$ of cells undergoing cell cycle arrest in G0/G1. This synergistic effect was confirmed in a xenotransplantation model where combination therapy resulted in a pronounced inhibition of disease progression which occurred early and was durable. Subsequently, the authors interrogated differential gene expression profiles of SKM1 cells treated with either drug alone versus the combination of APR-246 and azacitidine. As expected, Gene Set Enrichment Analysis (GSEA) and DAVID analyses of APR246 treated cells showed robust induction of p53-target genes including CDKN1A, CASP1, BAX and FAS, which was confirmed by reverse transcription real-time quantitative polymerase chain reaction (RT-qPCR), resulting in activation of an early apoptotic program. Furthermore, GSEA analysis of "synergistic only" genes (i.e. genes differentially expressed only with combination treatment) 


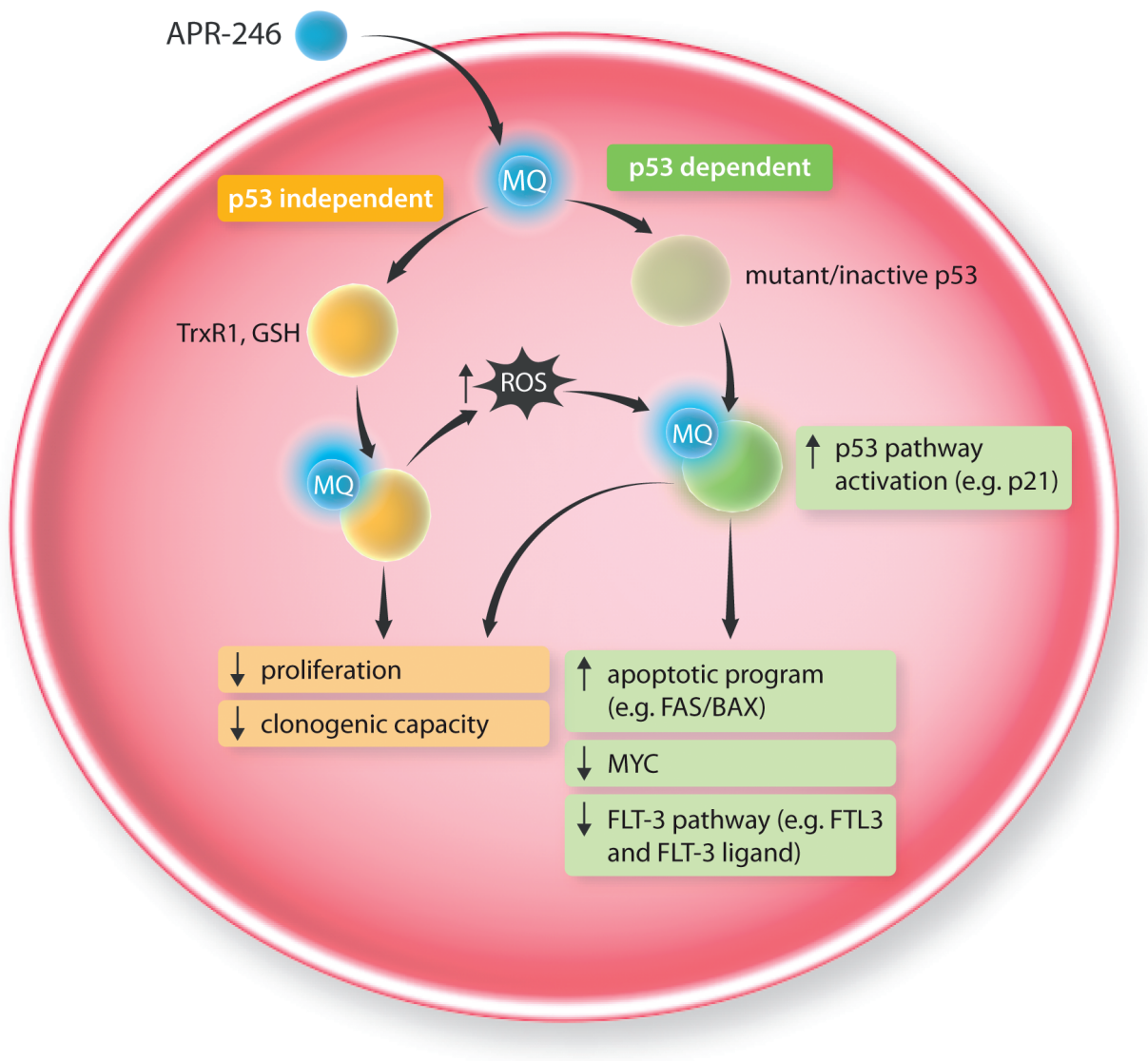

Figure 1. Mechanisms of synergy with APR-246 and azacitidine in TP53 mutant myelodysplastic syndromes (MDS) / acute myeloid leukemia (AML). GSH: glutathione; MQ: methylene quinuclidinone; ROS: reactive oxygen species; wt: wild type; TrxR1: thioredoxin reductase 1 FLT-3: fms like tyrosine kinase 3.

identified activation of p53 pathway, induction of apoptosis, and downregulation of MYC expression, thus functionally demonstrating restoration of wild-type p53 function. Notably, transcriptome analysis with confirmation by RT-qPCR also identified a novel synergistic mechanism of FLT3 pathway downregulation. Importantly, the inhibition of cell proliferation with combination therapy could be overcome in a dose-dependent fashion in the presence of FLT3 ligand, highlighting a novel therapeutic mechanism of APR-246 that could potentially be exploited in combination with FLT3 inhibitors in future clinical study.

Of importance, synergy was most robust in the presence of TP53 missense mutations where there is accumulation of misfolded p53 protein, strongly supporting the primary mechanism of APR-246. However, APR-246 also has $\mathrm{p} 53$-independent function via $\mathrm{MO}$ binding to thioredoxin reductase and glutathione, leading to depletion of glutathione and accumulation of reactive oxygen species (ROS), which can feed forward p53 activation (Figure 1). ${ }^{18,19}$ Indeed, the authors also show synergy in TP53 knockout mutant cell lines where there is absence of p53, albeit with less synergy than in the missense mutant model. Accordingly, there was significant enrichment of ROS-induced genes with APR-246 treatment. The authors also show data whereby both cell proliferation and clonogenic capacity were strongly inhibited, both in the presence and absence of mutant p53 protein.

Perhaps the most compelling data regarding the synergy of APR-246 and azacitidine originates from the clinical activity in TP53 mutant MDS/AML patients, where recent data report an overall and complete remission rate of $87 \%$ and $53 \%$, respectively (clinicaltrials.gov identifier: NCT03072043). ${ }^{9}$ Similarly, preliminary results from a phase II study of APR-246 and azacitidine by the Groupe Francophone des Myélodysplasies (clinicaltrials.gov identifier: NCT03588078) showed comparable response rates. ${ }^{10}$ Accordingly, the US Food and Drug Administration has recently granted breakthrough therapy designation for the treatment of patients with TP53 mutant MDS with the combination of APR-246 and azacitidine and the randomized phase III study of APR-246 and azacitidine versus azacitidine is ongoing in MDS patients (clinicaltrials.gov identifier: NCT03745716). As TP53 mutations are strong drivers of negative outcomes in multiple hematologic malignancies, as exemplified by relapsed pediatric acute lymphoblastic leukemia, APR-246 may likely have more broad clinical implications including synergy with traditional cytotoxic agents, as has been recently described. ${ }^{20}$ Together, shedding light on the synergistic mechanisms underlying APR-246 and azacitidine therapy as presented in this study are critical to continue to advance this novel therapeutic option for patients with the poorest outcomes to traditional treatments.

\section{References}

1. Papaemmanuil E, Gerstung M, Malcovati L, et al. Clinical and biological implications of driver mutations in myelodysplastic syndromes. Blood. 2013;122(22):3616-3627.

2. Bejar R, Stevenson K, Abdel-Wahab O, et al. Clinical Effect of Point 
Mutations in Myelodysplastic Syndromes. N Engl I Med. 2011;364(26):2496-2506.

3. Hunter AM, Sallman DA. Current status and new treatment approaches in TP53 mutated AML. Best Pract Res Clin Haematol. 2019;32(2):134-144.

4. Sallman DA, Komrokji R, Vaupel C, et al. Impact of TP53 mutation variant allele frequency on phenotype and outcomes in myelodysplastic syndromes. Leukemia. 2016;30(3):666-673.

5. Haase D, Stevenson KE, Neuberg D, et al. TP53 mutation status divides myelodysplastic syndromes with complex karyotypes into distinct prognostic subgroups. Leukemia. 2019;33(7):1747-1758

6. Montalban-Bravo G, Kanagal-Shamanna R, Benton CB, et al. Genomic context and TP53 allele frequency define clinical outcomes in TP53-mutated myelodysplastic syndromes. Blood Adv. 2020;4(3):482-495

7. Bernard E, Nannya Y, Yoshizato T, et al. TP53 State Dictates Genome Stability, Clinical Presentation and Outcomes in Myelodysplastic Syndromes. Blood. 2019;134(Supplement_1):675675

8. Hunter AM, Sallman DA. Targeting TP53 Mutations in Myelodysplastic Syndromes. Hematol Oncol Clin North Am. 2020;34(2):421-440.

9. Sallman DA, DeZern AE, Garcia-Manero G, et al. Phase 2 Results of APR-246 and Azacitidine (AZA) in Patients with TP53 mutant Myelodysplastic Syndromes (MDS) and Oligoblastic Acute Myeloid Leukemia (AML). Blood. 2019;134(Supplement_1):676-676.

10. Cluzeau T, Sebert M, Rahmé R, et al. APR-246 Combined with Azacitidine (AZA) in TP53 Mutated Myelodysplastic Syndrome (MDS) and Acute Myeloid Leukemia (AML). a Phase 2 Study By the Groupe Francophone Des Myélodysplasies (GFM). Blood. 2019;134(Supplement_1):677-677.
11. Maslah N, Salomao N, Drevon L, et al. Synergistic effects of PRIMA1Met (APR-246) and Azacitidine in TP53-mutated myelodysplastic syndromes and acute myeloid leukemia. Haematologica. 2019; xxx

12. Boettcher S, Miller PG, Sharma R, et al. A dominant-negative effect drives selection of TP53 missense mutations in myeloid malignancies. Science. 2019;365(6453):599-604.

13. Lambert IM, Gorzov P, Veprintsev DB, et al. PRIMA-1 reactivates mutant p53 by covalent binding to the core domain. Cancer Cell. 2009;15(5):376-388.

14. Zhang Q, Bykov VJN, Wiman KG, Zawacka-Pankau J. APR-246 reactivates mutant p53 by targeting cysteines 124 and 277. Cell Death Dis. 2018;9(5):439.

15. Lehmann S, Bykov VI, Ali D, et al. Targeting p53 in vivo: a first-inhuman study with p53-targeting compound APR-246 in refractory hematologic malignancies and prostate cancer. J Clin Oncol 2012:30(29):3633-3639.

16. Deneberg S, Cherif H, Lazarevic V, et al. An open-label phase I dosefinding study of APR-246 in hematological malignancies. Blood Cancer J. 2016;6(7):e447.

17. Cluzeau T, Dubois A, Jacquel A, et al. Phenotypic and genotypic characterization of azacitidine-sensitive and resistant SKM1 myeloid cell lines. Oncotarget. 2014;5(12):4384-4391.

18. Bykov VJ, Zhang Q, Zhang M, Ceder S, Abrahmsen L, Wiman KG Targeting of Mutant p53 and the Cellular Redox Balance by APR-246 as a Strategy for Efficient Cancer Therapy. Front Oncol. 2016;6:21

19. Peng X, Zhang MQ, Conserva F, et al. APR-246/PRIMA-1MET inhibits thioredoxin reductase 1 and converts the enzyme to a dedicated NADPH oxidase. Cell Death Dis. 2013;4(10):e881.

20. Demir S, Boldrin E, Sun Q, et al. Therapeutic targeting of mutant p53 in pediatric acute lymphoblastic leukemia. Haematologica. 2020;105(1):170-181.

\section{Peripheral T-cell lymphoma diagnosis: building a molecular tool}

\section{Miguel A Piris ${ }^{1,2}$}

${ }^{1}$ Department of Pathology, Hospital Universitario, Fundación Jiménez Díaz and ${ }^{2} C I B E R O N C$, Madrid, Spain

E-mail: MIGUEL A PIRIS - mapirispinilla@gmail.com

doi:10.3324/haematol.2020.249052

T -cell lymphoma (TCL) has quite a poor probability of survival (around $25-30 \%$ of patients after 5 years), which contrasts with the progress that has recently been made in Hodgkin lymphoma and B-cell lymphoma. ${ }^{1-3}$ Some recently defined TCL types, such as anaplastic large-cell lymphoma (ALCL), have a better clinical outcome. However, the majority of cases diagnosed with peripheral T-cell lymphoma (PTCL) will eventually die of the disease, and in some specific tumor types, such as intestinal TCL, the prognosis is even more miserable.

Poor survival probability in this context is associated with serious difficulties in lymphoma diagnosis when using routine morphological and immunohistochemistry tools. PTCL classification involves division into multiple subtypes, typically of low frequency and with hazy distinctions (Figure 1). As a consequence, different studies coincide in achieving a very low rate of reproducibility in TCL diagnosis, especially in recognizing ALK-negative ALCL, and distinguishing between PTCL-not otherwise specified (NOS) and PTCL with TFH phenotype or angioimmunoblastic TCL. ${ }^{4}$

An important feature of this situation is that the relative frequencies of the tumor types are quite low, which makes it difficult to design and develop clinical trials, and this hampers the introduction of new drugs for PTCL therapy.
Nevertheless, these difficulties have inspired some research groups to provide essential information about the molecular basis of TCL pathogenesis, and to identify some attractive and challenging therapeutic targets..$^{5.9}$

Drieux and co-workers, ${ }^{10}$ in a joint project involving French, Belgian and Swiss hospitals, are now addressing the radical proposal that molecular diagnosis may give a more precise and reproducible way of classifying TCL cases. Using a technique applicable to paraffin-embedded tissue, they measure the expression of 20 genes, including 17 markers relevant to T-cell classification, one EpsteinBarr virus-related transcript, and frequently mutated variants of RHOA (G17V) and IDH2 (R172K/T). Selected genes allow the identification of several entities: TFH cells, the normal counterparts of angioimmunoblastic TCL; TH1 and TH2 phenotypes, which reflect the diversity of PTCL-NOS; T-regulatory cells, for distinguishing ATLL; the cytoxic markers, CD30 and ALK, for identifying ALCL; and CD56 and EBER1, to discriminate T/natural killer (NK)-cell lymphomas.

The results validate the solid basis of the currently used PTCL classification scheme, and highlight the similarity between angioimmunoblastic TCL and PTCL-TFH. The findings show a group of cases with simultaneous expression of TFH markers and TH2 (GATA3), and indicate that ALK-negative ALCL is a heterogeneous condition. Cases of PTCL-NOS appear to be extremely heterogeneous, 